\title{
Effects of Oral or Parenteral Vitamin D Supplementation on Kidney Function, Blood Concentrations of 25-Hydroxyvitamin D, and Parathyroid Hormone Levels
}

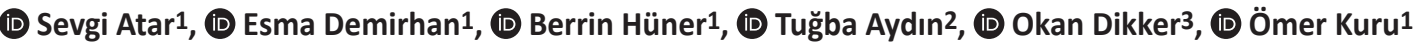 \\ 1 University of Health Sciences Turkey, Prof. Dr. Cemil Taşşıoğlu City Hospital, Clinic of Physical Therapy and Rehabilitation, Istanbul, Turkey \\ 2istanbul Physical Medicine Rehabilitation Training and Research Hospital, Clinic of Physical Therapy and Rehabilitation, Istanbul, Turkey \\ 3University of Health Sciences Turkey, Prof. Dr. Cemil Taşçıŏlu City Hospital, Clinic of Biochemistry, İstanbul, Turkey
}

\section{Abstract}

Objective: The aim of this study was to determine the effects of vitamin D supplementation on kidney function, blood concentrations of 25-hydroxyvitamin D, and parathyroid hormone levels.

Methods: The 25-hydroxyvitamin D levels of approximately 4,000 participants between the ages of 18 and 75 years were investigated retrospectively. Inclusion criteria included screening 25 -hydroxyvitamin $D<10 \mathrm{ng} / \mathrm{mL}$ at the local clinical laboratory. This study required the control of blood parameters at 2 and 6 weeks. Because of this, 120 patients were chosen and categorized according to treatment procedures. The first group of participants was prescribed a fixed dose of 300,000 IU oral vitamin D filled at local clinical pharmacies. The second group of participants was prescribed a fixed dose of 300,000 IU parenteral vitamin D filled at local clinical pharmacies. The last group of participants was prescribed a fixed dose of 50,000 IU once weekly oral vitamin D once weekly for six weeks filled at local clinical pharmacies. Vitamin D values and biochemical laboratory parameters were recorded and were compared between groups using statistical methods.

Results: The treatment regimens with 25-hydroxyvitamin D3 were divided equally among three patient groups $(n 1=40, n 2=40, n 3=40)$. The findings were that 25 -hydroxyvitamin $D$ levels changes in the first $(p=0.001)$ and third groups $(p=0.001)$ were greater than the second group $(p<0.01)$.

Conclusion: These effects occur with significant changes in oral vitamin D supplementation rather than when vitamin D is administered parenterally.

Keywords: Vitamin D, calcium, oral, parenteral

\section{INTRODUCTION}

Vitamin D is synthesized in the body with the effect of 90\%-95\% of sunlight. Besides, it can be dissolved in oils from the intake of dietary sources. It physically looks like some hormones such as testosterone, estrogen, cortisol, and others (1-3). During the last 2 decades, vitamin $D$ has been recognized for having very positive effects on the immune system and the cardiovascular system (4).
Vitamin D deficiency, which affects about one million people, is a public health problem. Intake from the diet is limited. It exists as cholecalciferol (D3) and ergocalciferol (D2) and is found in the diet mostly in fatty fish, such as salmon and egg yolks (5). Reasons for the deficiency include altitude, season, cultural malnutrition, clothing style, domestic lifestyle, skin pigmentation, tuberculosis, some drugs, and genetics (2). Deficiency can be restrained by low-cost intake (6). Although it 
does not have an exact definition, the level of 25-hydroxyvitamin $\mathrm{D}[25(\mathrm{OH}) \mathrm{D}]$ is a standard parameter used to indicate accurate vitamin D levels (7). As a treatment, D3 is much more suitable than D2. Moreover, treatment can be taken for daily support (8) because low circulating concentrations of $25(\mathrm{OH}) \mathrm{D}$ are common, and a deficiency in $25(\mathrm{OH}) \mathrm{D}$ may contribute to adverse health outcomes. Despite widespread use, the effects of vitamin D supplements on downstream vitamin D metabolism are unclear. According to the literature, vitamin D filled at local clinical pharmacies at 50,000 IU once weekly for 6-8 weeks can achieve circulating concentrations of $25(\mathrm{OH}) \mathrm{D}$ of at least 30 $\mathrm{ng} / \mathrm{mL}(9)$.

According to studies, vitamin D deficiency's treatment is not clear. Oral or parenteral methods are controversial (10). Also, the information regarding the bioavailability of parenteral treatment is limited. Because of this, our study is based on the circulating concentrations of $25(\mathrm{OH}) \mathrm{D}$, parathyroid hormone (PTH), and kidney function (spot urine sample).

\section{METHODS}

This study was approved by the institutional review boards of Okmeydani Training and Research Hospital in 2019 (approvel no: 02.04.2019-1227). The study was initiated before adopting the expanded definition of a clinical trial by the International Committee of Medical Journal Editors in 2018. It was considered at the time to be a detailed physiologic study rather than a clinical trial. For this reason, it was not prospectively entered into a clinical trials registry. Between 2018 and 2019, approximately 4,000 participants between 18 and 75 were recruited from the Okmeydani Training and Research Hospital outpatient physiotherapy clinics. Their $25(\mathrm{OH}) \mathrm{D}$ levels were investigated retrospectively. All potential candidates showing interest in the study were invited to attend a screening visit, at which eligibility for inclusion was assessed, and informed consent was obtained. Inclusion criteria included screening $25(\mathrm{OH}) \mathrm{D}<10 \mathrm{ng} / \mathrm{mL}$ at the local clinical laboratory. This study required controlling blood parameters [glucose, urea, creatinine, calcium (Ca), potassium, alp, PTH] at 2 and 6 weeks was needed. Because of this, 120 patients were chosen and categorized according to treatment procedures. The exclusion criteria are hypercalcemiahypercalciuria, chronic kidney failure, kidney stones, using any drugs that affect vitamin D and bone metabolism, geographic information systems disease (malabsorption), osteoporosis treatment, malignancy, diabetes mellitus, hyperthyroidism, cancer, rheumatoid arthritis, chronic drug use, and alcohol abuse.
The first group of participants was prescribed a fixed dose of 300,000 oral vitamin D filled at local clinical pharmacies. The second group of participants was prescribed a fixed dose of 300,000 parenteral vitamin D filled at local clinical pharmacies. The last group of participants was prescribed a fixed dose of 50,000 IU oral vitamin D once weekly for 6 weeks, filled at local clinical pharmacies.

Measurements of laboratory tests: Glucose, urea, creatinine, phosphorus, and Ca tests were measured with the calorimetric method, PTH, and 25-hydroxyvitamin D3 values were measured with chemiluminescence method in an autoanalyser (BeckmanCoulter TM, AU 5800 model, USA).

\section{Statistical Analyses}

Descriptive statistics were used to compare baseline participant characteristics. The number cruncher statistical system 2007 (Kaysville, Utah, USA) was used for these analyses. The ShapiroWilk test was used to assess the normality of outcomes distribution. The statistical evaluation of the three groups involved applying the One-Way ANOVA test and Bonferroni test that were chosen for parametric variables. The other variables were assessed with the Kruskal-Wallis test and BonferroniDunn test. The Wilcoxon signed-rank test was used for the first and sixth-week comparisons of variables that did not show a normal distribution. The repeated measures test was used for the first, second, and sixth-week comparisons of normally distributed variables, and the Bonferroni test was used for binary comparisons. The Friedman test was used for the first, second, and sixth-week comparisons of variables that did not show a normal distribution. The Bonferroni-Dunn test was used for binary comparisons. Qualitative data comparisons were made using Pearson's chi-square test, and the Fisher-Freeman-Halton test was used. Spearman Rho and Pearson correlation tests were used to determine correlations between the variables tested. For all the calculations in the study, a $p$ value less than 0.05 was considered statistically significant.

\section{RESULTS}

In our study of 120 patients, vitamin D was administered intermittently in $33.3 \%(n=40)$ of the cases, by intramuscular (IM) in $33.3 \%(n=40)$, by mouth in $33.3 \%(n=40)$. Women comprised $79.2 \%(n=95)$ of the cases and men comprised $20.8 \%$ $(n=25)$ of the cases. The body mass index scale was between 17.2 and 42.2 with an average of $26.79+4.36$. Regarding educational level, 5.8\% $(n=7)$ were illiterate, $8.3 \%(n=10)$ were literate, $62.5 \%$ $(n=75)$ graduated from primary school, $15.1 \%(n=18)$ graduated from high school, and $8.3 \%(n=10)$ graduated from a university. 
The dependency on the duration of physical activity was observed in: $24.2 \%(n=29)$ less than an hour per week, 34.1\% $(n=41) 1-2$ hours per week, $4.2 \%(n=5) 4-7$ hours per week, and $7.5 \%(n=9) 7$ hours per week.

Concerning sunbathing, $70.0 \%(\mathrm{n}=84)$ less than an hour per week, $14.2 \%(n=17) 1-2$ hours per week, 5.8\% $(n=7) 2-4$ hours per week, $4.2 \%(n=5) 4-7$ hours per week, and $5.8 \%(n=7) 7$ hours per week.

Regarding Ca intake: $14.2 \%(n=17)$ of cases do not take any at all; the rest of them take Ca.

Concerning smoking: $21.7 \%(n=26)$ of cases have a smoking habit, the rest do not use tobacco.

When asked about clothing style, answers were: 1.7\% $(n=2)$ local style, 43.3\% ( $n=52)$ modern style, and 55\% ( $n=66)$ closed style.

For all the calculations in the study, a $p$ value less than 0.05 was considered statistically significant. Educational level $(p=0.002$; $p<0.01$ ), physical activity frequencies $(p=0.004 ; p<0.01)$, and $\mathrm{Ca}$ intake are statistically significant.
Evaluation of the level of vitamin D: According to the groups, there was no statistically significant difference $(p=0.995 ; p>0.05)$.

According to the groups, the sixth-week measurement of vitamin $\mathrm{D}$ is statistically significant $(\mathrm{p}=0.001)$. The correlation between the groups' vitamin D intake by oral, intermittent, and IM is statistically significant $(p<0.05)$. Oral and intermittent intake forms have an advantage on intake.

All three groups sixth-week measurements are statistically significant (intermittent $p=0.001$, IM $p=0.001$, oral $p=0.001$ ).

The evaluation of the level of PTH: According to the groups, there was no statistically significant difference between the first $(p=0.883)$ and sixth-week levels $(p=0.358)$.

All three groups' sixth-week levels decrease is statistically significant (intermittent $p=0.01$, IM $p=0.002$, oral $p<0.01$ ).

There is not any differences between groups sixth-week results $(p=0.197 ; p>0.05)($ Table 1$)$.

According to the groups, first and sixth-week circulating Ca levels changes are not significant as statistically $(p>0.05)$.

\section{Table 1. According to vitamin D groups, evaluation of circulating concentrations of PTH, vitamin D, and phosphorus levels}

\begin{tabular}{|c|c|c|c|c|c|}
\hline & & \multicolumn{3}{|c|}{ Type of vitamin D intake } & \multirow[t]{2}{*}{$b_{p}$} \\
\hline & & Intermittent $(\mathrm{n}=\mathbf{4 0})$ & IM $(n=40)$ & Oral $(n=40)$ & \\
\hline \multirow{2}{*}{$1^{\text {st }}$ vitamin $D$} & Min-max (median) & $2-10(8.3)$ & $1.4-10(8.2)$ & $2.3-10(8)$ & \multirow{2}{*}{0.995} \\
\hline & Average \pm SD & $7.66 \pm 2.05$ & $7.64 \pm 2.02$ & $7.61 \pm 2.04$ & \\
\hline \multirow{2}{*}{$6^{\text {th }}$ week } & Min-max (median) & $19.7-80.2(43.8)$ & $14-57.5(25)$ & 23.9-77 (45.5) & \multirow{2}{*}{$0.001^{* *}$} \\
\hline & Average \pm SD & $43.65 \pm 11.79$ & $25.06 \pm 8.57$ & $46.32 \pm 11.49$ & \\
\hline & ep & $0.001 * *$ & $001 * *$ & $0.001^{* *}$ & \\
\hline \multirow{2}{*}{ Difference } & Min-max (median) & $11.2-73.9(37)$ & $6.6-49.7(16.7)$ & $13.9-69.9(36.4)$ & \multirow{2}{*}{$0.001^{* *}$} \\
\hline & Average \pm SD & $35.99 \pm 11.54$ & $17.42 \pm 8.71$ & $38.71 \pm 11.87$ & \\
\hline \multirow{2}{*}{$1^{\text {st }}$ PTH } & Min-max (median) & $22.8-125.4(55)$ & $20.9-114(60)$ & $22-124(53.6)$ & \multirow{2}{*}{0.883} \\
\hline & Average \pm SD & $58.05 \pm 22.52$ & $59.66 \pm 21.29$ & $58.80 \pm 22.83$ & \\
\hline \multirow{2}{*}{$6^{\text {th }}$ week } & Min-max (median) & $18.1-79.4(47.6)$ & $18.8-70(41.5)$ & 30.9-89.1 (45.5) & \multirow{2}{*}{0.358} \\
\hline & Average \pm SD & $45.61 \pm 14.39$ & $43.48 \pm 13.40$ & $48.00 \pm 11.84$ & \\
\hline & $\mathrm{ep}_{\mathrm{p}}$ & $0.001^{* *}$ & $0.001^{* *}$ & $0.002^{* *}$ & \\
\hline \multirow{2}{*}{ Difference } & Min-max (median) & $-76.2-22.5(-7.9)$ & $-60.1-15.7(-14)$ & $-70-24.9(-4.7)$ & \multirow{2}{*}{0.197} \\
\hline & Average \pm SD & $-12.44 \pm 18.98$ & $-16.18 \pm 16.95$ & $-10.80 \pm 20.04$ & \\
\hline \multirow{2}{*}{$1^{\text {st }}$ phosphorus } & Min-max (median) & $2.6-4.6(3.6)$ & $2.5-8.5(3.6)$ & $2.7-4.6(3.6)$ & \multirow{2}{*}{0.626} \\
\hline & Average \pm SD & $3.63 \pm 0.50$ & $3.63 \pm 0.93$ & $3.63 \pm 0.44$ & \\
\hline \multirow{2}{*}{$6^{\text {th }}$ week } & Min-max (median) & 3-4.9(3.5) & 3-5 (3.8) & $3.1-4.6(3.8)$ & \multirow{2}{*}{0.149} \\
\hline & Average \pm SD & $3.64 \pm 0.51$ & $3.76 \pm 0.41$ & $3.80 \pm 0.39$ & \\
\hline & $e_{p}$ & 0.877 & $0.010^{*}$ & $0.010^{*}$ & \\
\hline \multirow{2}{*}{ Difference } & Min-max (median) & $-1-0.9(0)$ & $-4.6-1.3(0.3)$ & $-0.7-0.9(0.2)$ & \multirow{2}{*}{0.194} \\
\hline & Average \pm SD & $0.01 \pm 0.55$ & $0.12 \pm 0.91$ & $0.17 \pm 0.41$ & \\
\hline
\end{tabular}


second circulating Ca levels are statistically significant to each other $(p=0.009)$. Intake of vitamin $D$ by oral is much more effective than by IM intake $(p=0.007)$.

Second measurements are significant to each other $(p=0.003)$. Intake of vitamin $\mathrm{D}$ by oral is much more effective than by IM intake $(p<0.05)$.

The evaluation of the Ca level in urine: According to the groups, first and sixth-week urine Ca levels changes are not significant as statistically $(p>0.05)$.

Sixth-week circulating Ca levels are statistically significant to each other. Intake of vitamin D by oral is much more effective than by IM intake $(p=0.049)$ (Table 2$)$.
The evaluation of $\mathrm{Ca} / \mathrm{Cre}$ ratio in urine: According to groups, there is not any significant difference between first, second, and sixth measurement $(p=0.945 ; p=0.871 ; p=0.972)$.

Regarding the difference between measurement times, there is no statistically significant difference (Table 3 ).

\section{DISCUSSION}

Although the effects of vitamin D and deficiency are well known, there is no definite opinion on the effectiveness of the forms to be used in treatment. There are many studies on the effectiveness and ease of use of oral or parenteral forms, and each reported different results (8-11).

\begin{tabular}{|c|c|c|c|c|c|}
\hline & & \multicolumn{3}{|l|}{ Vitamin D intake } & \multirow[t]{2}{*}{$b_{p}$} \\
\hline & & Intermittent $(n=40)$ & $\mathrm{IM}(\mathrm{n}=40)$ & Oral $(n=40)$ & \\
\hline $1^{\text {st }}$ circulating $\mathrm{Ca}$ & Average \pm SD & $9.58 \pm 0.36$ & $9.60 \pm 0.42$ & $9.57 \pm 0.38$ & $\mathrm{a} 0.949$ \\
\hline $2^{\text {nd }}$ circulating $\mathrm{Ca}$ & Min-max (median) & 9-10.8 (9.6) & $7-10.3(9.5)$ & 8.9-10.7 (9.7) & $\mathrm{a} 0.009 * *$ \\
\hline \multirow{2}{*}{$6^{\text {th }}$ week circulating $\mathrm{Ca}$} & Min-max (median) & 8.8-10.5 (9.6) & 8.8-10.2 (9.5) & 8.4-10.3 (9.7) & \multirow{2}{*}{$\mathrm{a} 0.157$} \\
\hline & Average \pm SD & $9.53 \pm 0.40$ & $9.47 \pm 0.38$ & $9.63 \pm 0.33$ & \\
\hline & $f_{p}$ & 0.709 & 0.117 & $0.002^{* *}$ & \\
\hline Difference (2nd-1st) & Min-max (median) & $-0.9-1.1(-0.1)$ & $-2.4-0.8(-0.1)$ & $-0.5-1.1(0.2)$ & $0.003^{* *}$ \\
\hline \multirow{2}{*}{ Difference (6 $\left.6^{\text {th }}-2^{\text {nd }}\right)$} & Min-max (median) & $-1.3-0.8(-0.1)$ & $-0.9-1.9(0)$ & $-1.2-0.6(-0.1)$ & \multirow{2}{*}{0.518} \\
\hline & Average \pm SD & $-0.05 \pm 0.46$ & $0.03 \pm 0.54$ & $-0.12 \pm 0.33$ & \\
\hline \multirow{2}{*}{ First urine $\mathrm{Ca}$} & Min-max (median) & $3.1-22.7(9)$ & 3.9-19.8 (8.1) & $3.2-21.2(7.6)$ & \multirow{2}{*}{0.984} \\
\hline & Average \pm SD & $9.21 \pm 5.08$ & $9.22 \pm 4.79$ & $9.05 \pm 4.9$ & \\
\hline \multirow{2}{*}{$2^{\text {nd }}$ urine $\mathrm{Ca}$} & Min-max (median) & $4.2-23.4(9.8)$ & $1.2-29.8(11)$ & 3.1-31.7 (11.4) & \multirow{2}{*}{0.944} \\
\hline & Average \pm SD & $11.57 \pm 5.55$ & $12.17 \pm 7.62$ & $11.87 \pm 7.38$ & \\
\hline \multirow{2}{*}{$6^{\text {th }}$ week urine $\mathrm{Ca}$} & Min-max (median) & $1.6-30(10)$ & $4.1-46.4(9.7)$ & $3.4-23.8(8.8)$ & \multirow{2}{*}{0.728} \\
\hline & Average \pm SD & $10.33 \pm 6.19$ & $12.09 \pm 8.33$ & $11.41 \pm 6.72$ & \\
\hline Difference (6 $\left.6^{\text {th }}-2^{\text {nd }}\right)$ & Average \pm SD & $-1.24 \pm 6.95$ & $-0.08 \pm 6.53$ & $-0.45 \pm 8.09$ & 0.805 \\
\hline
\end{tabular}


In recent years, research on vitamin $D$ as randomized controlled studies on vitamin D replacement has increased. Between 2003 and 2017, there was a higher increase in publications scanned as "vitamin D supplementation" in PubMed compared with all studies on vitamin D. It is estimated that this number will increase even more with more than 100 randomized controlled vitamin $D$ replacement studies registered in the ClinicalTrals.gov system (11).

We planned this study based on this increase. This study aimed to evaluate the effects of oral, intermittent oral, and parenteral $25(\mathrm{OH})$ D replacement on vitamin D blood levels, PTH levels, and kidney function.

In this study, the increase in the sixth-week measurements was statistically significant compared with the first measurements of vitamin D in all three groups. Intermittent oral and single dose orally administered vitamin D measurements at six weeks between groups were higher than those receiving vitamin D intramuscularly. However, there was no statistically significant difference between the measurements of the groups taking intermittent oral and single dose oral vitamin D. Gupta et al. (12) investigated the effect of oral (5 weeks of 60,000 IU) and IM (300,000 IU) vitamin D replacement in the treatment of vitamin $D$ deficiency. They detected a significant increase in vitamin $D$ in both groups at the end of the sixth-week.

Whyte et al. (13) reported that oral or intravenous (IV) forms showed a definite increase in a week in their study comparing different vitamin D forms in healthy adults. This increase in IM and subcutaneous forms occurred only in the seventh week, and vitamin D levels decreased in oral or IV form in the same period. In our study, oral forms had a more significant increase at the end of the sixth-week than those who used IM forms. It may be because this study was conducted using healthy adults might have caused a difference in bioavailability times between our study when compared with those with vitamin D deficiency.

In their study, Zabihiyeganeh et al. (14) compared vitamin D administered as a single dose 300,000 IU IM injection and six doses of 50,000 IU oral D3 vitamin D in the treatment of hypovitaminosis $\mathrm{D}$. In the $3^{\text {rd }}$ month, the $25(\mathrm{OH}) \mathrm{D}$ vitamin level was significantly higher than that given by the oral route, similar to our study. However, Zabihiyeganeh et al. (14) reported that the sixth-month measurements were similar.

Tellioglu et al. (15) evaluated 116 nursing home residents aged 65 and over. Of these, 600,000 IU D3 were administered IM or orally to 66 people whose $25(\mathrm{OH}) \mathrm{D}$ level was found below $<30 \mathrm{ng} / \mathrm{mL}$. They evaluated the biochemical results at 6 and 12 weeks. Although the results at 6 weeks were consistent with our study results, at 12 weeks, the increase in the $25(\mathrm{OH}) \mathrm{D}$ level of the IM group was significantly higher than the oral group.

PTH levels are expected to be high in patients with vitamin D deficiency (16). In our study, the first measurements in the intermittent oral group $(58.05 \pm 22.52 \mathrm{pg} \mathrm{mL})$ were not significantly higher than in the IM group $(59.66 \pm 21.29 \mathrm{pg} / \mathrm{mL})$ and in the dose oral group $(58.80 \pm 22.83 \mathrm{pg} / \mathrm{mL})$. In their study, Sahota et al. (17) reported that vitamin D deficiency did not increase PTH value in some cases. However, PTH levels at six weeks in all groups in our study were found to be significantly

Table 3. According to vitamin D groups, evaluation of urine concentrations of calcium/creatinine level

\begin{tabular}{|c|c|c|c|c|c|}
\hline & & \multicolumn{3}{|l|}{ Vitamin D intake } & \multirow[t]{2}{*}{$b_{p}$} \\
\hline & & Intermittent $(n=40)$ & $\mathrm{IM}(\mathrm{n}=40)$ & Oral $(n=40)$ & \\
\hline $1^{\text {st }} \mathrm{Ca} / \mathrm{Cre}$ & Min-max (median) & $0-0.1(0.04)$ & $0.01-0.09(0.04)$ & 0-0.1 (0.05) & 0.945 \\
\hline \multirow{2}{*}{$2^{\text {nd }} \mathrm{Ca} / \mathrm{Cre}$} & Min-max (median) & 0.01-0.1 (0.05) & $0.01-0.1(0.04)$ & $0-0.1(0.05)$ & \multirow{2}{*}{0.871} \\
\hline & Average \pm SD & $0.05 \pm 0.03$ & $0.05 \pm 0.03$ & $0.05 \pm 0.03$ & \\
\hline \multirow{2}{*}{$6^{\text {th }} \mathrm{Ca} / \mathrm{Cre}$} & Average \pm SD & $0.05 \pm 0.03$ & $0.05 \pm 0.03$ & $0.05 \pm 0.03$ & 0.972 \\
\hline & $g_{p}$ & 0.684 & 0.282 & 0.921 & \\
\hline \multirow{2}{*}{ Difference $\left(2^{\text {nd }}-1\right.$ st $)$} & Min-max (median) & $-0.07-0.09(0)$ & $-0.07-0.08(0)$ & \begin{tabular}{|l}
$-0.07-0.07(0)$ \\
\end{tabular} & \multirow{2}{*}{0.890} \\
\hline & Average \pm SD & $0 \pm 0.04$ & $0 \pm 0.04$ & $0 \pm 0.03$ & \\
\hline Difference ( $\left.6^{\text {th }}-1^{\text {st }}\right)$ & Min-max (median) & $-0.07-0.07(0)$ & $-0.07-0.08(0)$ & $-0.07-0.07(0)$ & 0.937 \\
\hline Difference $\left(6^{\text {th }}-2^{\text {nd }}\right)$ & Average \pm SD & $0 \pm 0.04$ & $0 \pm 0.04$ & $0 \pm 0.04$ & 0.657 \\
\hline
\end{tabular}

${ }^{b}$ Kruskal-Wallis test, gFriedman test, Ca: Calcium, Cre: Creatinine, SD: Standard deviation, IM: Intramuscular, Min: Minimum, Max: Maximum 
lower than the first measurements. There was no statistically significant difference between the groups regarding changes in the first- and sixth-week measurements. Leventis and Kiely (18) found a decrease in PTH levels because of 12 weeks of 300,000 IU IM and oral vitamin D treatment.

A significant difference was detected between the groups in the second-week blood Ca results after vitamin D support in our study. The change in the group receiving oral vitamin D was found to be significantly higher than the groups receiving vitamin $D$ intermittently $(p=0.044)$ and with the IM method $(p=0.004 ; p<0.05)$.

An increase in blood Ca values was detected in the second-week measurements in all three groups. There was a statistically significant difference between the Ca measurements in the blood in the second-week, according to the groups $(p=0.009 ; p<0.01)$. The measurements of the group receiving vitamin $\mathrm{D}$ orally are higher than the group receiving vitamin D with the IM method $(p=0.007 ; p<0.01)$. No statistically significant difference was found in other binary comparisons ( $p>0.05)$. Blood Ca changes in the sixth-week measurements according to the first measurements, changes in the sixth-week measurements according to the secondweek measurements were not statistically significant $(p>0.05)$. In the treatment of vitamin D deficiency, blood Ca elevation is seen because of increased Ca absorption from the intestine and increased bone metabolism (19).

Spot Urine $\mathrm{Ca} / \mathrm{Kr}$ ratio is a simple, low-cost, and reliable method used to calculate Ca excretion due to difficulties in 24-hour urine Ca level. In our study, we also used spot urine $\mathrm{Ca} / \mathrm{Kr}$ excretion indices to determine hypercalciuria. In our study, values below 0.14 were normal, and values above were considered hypercalciuria. No patients had hypercalciuria. Leaf et al. (20) investigated the effect of vitamin D replacement on urinary Ca excretion by administering 50,000 IU D2 orally for eight weeks to patients with $\mathrm{D}$ hypovitaminosis with a history of nephrolithiasis. In their evaluation during the eighth week, they reported that, despite a significant increase in $25(\mathrm{OH}) \mathrm{D}$ vitamin levels, the mean 24-hour urinary Ca excretion did not change. Similarly, there was a significant increase in $25(\mathrm{OH}) \mathrm{D}$ levels in all three groups in our study, but no significant increase in mean spot urine Ca values.

\section{Study Limitations}

Our study has some limitations. The main limitations are that it is a retrospective study and the recorded follow-up times are relatively short. A longer follow-up period might contribute to a more meaningful evaluation of the effect of $25(\mathrm{OH}) \mathrm{D}$ levels and the impact of IM vitamin D treatment. It was observed that all three forms of administration are quite effective and safe in treating hypovitaminosis $\mathrm{D}$. Although oral therapy has been shown to increase the level of vitamin D significantly in our study, the mode of treatment may depend on patient selection and compliance.

\section{CONCLUSION}

It appears that inadequate vitamin D deficiency treatment leads to serious health problems, but treatment costs are relatively low. We think that prospective studies with high case numbers demonstrating the effectiveness of different forms of treatment will be beneficial.

\section{Ethics}

Ethics Committee Approval: Okmeydani Training and Research Hospital in 2019 (approvel no: 02.04.2019-1227).

Informed Consent: Informed consent was obtained.

Peer-review: Externally and internally peer-reviewed.

\section{Authorship Contributions}

Surgical and Medical Practices: S.A., O.D., Concept: S.A., Design: S.A., E.D., B.H., T.A., O.D., Ö.K., Data Collection or Processing: S.A., E.D., B.H., Analysis or Interpretation: S.A., E.D., B.H., O.D., Ö.K., Literature Search: S.A., T.A., Writing: S.A.

Conflict of Interest: No conflict of interest was declared by the authors.

Financial Disclosure: The authors declared that this study received no financial support.

\section{REFERENCES}

1. Sassi F, Tamone C, D’Amelio P. Vitamin D: Nutrient, Hormone, and Immunomodulator. Nutrients 2018;10:1656.

2. Moretti R, Morelli ME, Caruso P. Vitamin D in Neurological Diseases: A Rationale for a Pathogenic Impact. Int J Mol Sci 2018;19:2245.

3. Öngen B, Kabaroğlu C, Parıldar Z. Biochemical and Laboratory Evaluation of Vitamin D. Türk Klinik Biyokimya Derg 2008;6:23-31.

4. Galior K, Grebe S, Singh R. Development of Vitamin D Toxicity from Overcorrection of Vitamin D Deficiency: A Review of Case Reports. Nutrients 2018:10:953.

5. Holick MF. High prevalence of vitamin D inadequacy and implications for health. Mayo Clin Proc 2006;81:353-73.

6. Nair R, Maseeh A. Vitamin D: The "sunshine" vitamin. J Pharmacol Pharmacother 2012;3:118-26.

7. Institute of Medicine, Standing Committee on the Scientific Evaluation of Dietary Reference Intakes, Food and Nutrition Board. Vitamin D. In: Dietary Reference Intakes for Calcium, Phosphorus, Magnesium, Vitamin D, and Fluoride. Washington, DC: National Academies Pr; 1997. 
8. Tripkovic L, Lambert H, Hart K, Smith CP, Bucca G, Penson S, et al. Comparison of vitamin D2 and vitamin D3 supplementation in raising serum 25-hydroxyvitamin D status: a systematic review and metaanalysis. Am J Clin Nutr 2012;95:1357-64.

9. Dawson-Hughes B, Heaney RP, Holick MF, Lips P, Meunier PJ, Vieth R. Estimates of optimal vitamin D status. Osteoporos Int 2005;16:713-6.

10. Francis RM, Aspray TJ, Bowring CE, Fraser WD, Gittoes NJ, Javaid MK, et al. National Osteoporosis Society practical clinical guideline on vitamin D and bone health. Maturitas 2015;80:119-21.

11. Scragg R. Emerging Evidence of Thresholds for Beneficial Effects from Vitamin D Supplementation. Nutrients 2018;10:561.

12. Gupta N, Farooqui KJ, Batra CM, Marwaha RK, Mithal A. Effect of oral versus intramuscular Vitamin D replacement in apparently healthy adults with Vitamin D deficiency. Indian J Endocrinol Metab 2017;21:131-6.

13. Whyte MP, Haddad JG Jr, Walters DD, Stamp TC. Vitamin D bioavailability: serum 25-hydroxyvitamin D levels in man after oral, subcutaneous, intramuscular, and intravenous vitamin D administration. J Clin Endocrinol Metab 1979;48:906-11.

14. Zabihiyeganeh M, Jahed A, Nojomi M. Treatment of hypovitaminosis D with pharmacologic doses of cholecalciferol, oral vs intramuscular; an open labeled RCT. Clin Endocrinol (Oxf) 2013;78:210-6.
15. Tellioglu A, Basaran S, Guzel R, Seydaoglu G. Efficacy and safety of high dose intramuscular or oral cholecalciferol in vitamin $D$ deficient/ insufficient elderly. Maturitas 2012;72:332-8.

16. Emel T, Doğan DA, Erdem G, Faruk O. Therapy strategies in vitamin D deficiency with or without rickets: efficiency of low-dose stoss therapy. J Pediatr Endocrinol Metab 2012;25:107-10.

17. Sahota O, Gaynor K, Harwood RH, Hosking DJ. Hypovitaminosis D and 'functional hypoparathyroidism'-the NoNoF (Nottingham Neck of Femur) study. Age Ageing 2001;30:467-72.

18. Leventis P, Kiely PD. The tolerability and biochemical effects of highdose bolus vitamin D2 and D3 supplementation in patients with vitamin D insufficiency. Scand J Rheumatol 2009;38:149-53.

19. Blunt JW, Tanaka Y, DeLuca HF. The biological activity of 25-hydroxycholecalciferol, a metabolite of vitamin D3. Proc Natl Acad Sci U S A 1968;61:717-8.

20. Leaf DE, Korets R, Taylor EN, Tang J, Asplin JR, Goldfarb DS, et al. Effect of vitamin $\mathrm{D}$ repletion on urinary calcium excretion among kidney stone formers. Clin J Am Soc Nephrol 2012;7:829-34. 